\title{
Is Surgery for an FNA proven Warthin's Tumor Passé?
}

Nir Hirshoren ${ }^{1 *}$, Jonathan Cohen ${ }^{1}$, Tzahi Neuman², Jeffrey M. Weinberger ${ }^{1}$ and Ron Eliashar ${ }^{1}$

\begin{abstract}
Background: Warthin tumor is a common benign salivary gland lesion. Its optimal treatment mode is controversial. We analyze the accuracy of Warthin tumor pre-operative fine-needle aspiration (FNA)-based diagnosis and reassess the treatment paradigm. The risk of missing malignant tumors, falsely demonstrating a cytological benign Warthin tumor in the pre-operative FNA, is to be explored.

Methods: Retrospective cohort study in a tertiary university hospital. Data on all parotidectomy cases between 1996 and 2010 ( $n=238$ ) was analyzed. Cytological accuracy was calculated in 2 different opposing ways. First, we considered cases with a correct pre-operative identification of histologically proven Warthin tumors as true positives. The second assessment was done by gathering all cytological examinations suggesting a Warthin tumor, which were later excised. The histological identification of a malignant tumor was considered as a false negative.

Results: FNA was found to be accurate in 48 out of the 61 patients who had a final pathologic diagnosis of Warthin tumor, i.e. a $78.7 \%$ sensitivity. In the second step, there were 50 FNA's suggesting a Warthin tumor, One case turned out to be malignant: a low-grade muco-epidermoid carcinoma. The negative predictive value was therefore $98 \%$.

Conclusions: The overall accuracy of FNA suggesting Warthin tumor in a tertiary university hospital is high. We offer, in selected patients, observation as a reasonable alternative treatment to surgery. However, we also advise a repeat ultrasound-guided FNA to confirm the initial diagnosis.
\end{abstract}

\section{Background}

Warthin tumor (papillary cystadenoma lymphomatosum) is the second most common benign neoplasm of the parotid gland. Only rare cases demonstrate malignant transformation, constituting $0.3 \%$ of tumors [1]. Surgical treatment is considered the treatment of choice in most patients [2]. Surgical treatment is preferred not because of the tumor's aggressiveness, but rather because of uncertainty regarding the preoperative cytological accuracy. Lately, some surgeons have challenged this assumption, advocating conservative, strict follow-up in place of surgery $[3,4]$ The option of conservative treatment is appealing, since surgery clearly carries risks such as permanent facial nerve palsy. Moreover, Warthin tumor is more common when the patient is in his 50's or 60's, and the risk of general anesthesia may be high. At our hospital, FNA may be performed with or without ultrasonographic (US) guidance. Typically, US-guided FNA is performed in borderline clinical cases and in cases in which a previous FNA result was inconclusive. Our hospital policy obligates that only an experienced cytopathologist analyses all cytological specimens.

Currently, there is a paucity of data concerning FNA accuracy in Warthin tumors. Although most previous studies analyzed Warthin tumor patients who underwent a pre-operative FNA [5-8], the risk of missing malignant tumors, falsely demonstrating a cytological benign Warthin tumor in the pre-operative FNA, is still slight. We present our experience in managing Warthin tumor and the accuracy of a pre-operative FNA-based diagnosis.

Correspondence: drnir@hadassah.org.il

${ }^{1}$ Department of Otolaryngology/Head \& Neck Surgery

Hadassah Ein-Kerem, Jerusalem, Israel.

Full list of author information is available at the end of the article

\section{Materials \& Methods}

The study was performed under the approval of the local Internal Review Board (Helsinki Committee).

\section{Patients}

Data on all parotidectomy cases treated in our department between 1996 and 2010 ( $n=238$ ) was retrospectively analyzed (Figure 1). Seventy six patients had a final post-operative histological diagnosis of Warthin tumor. Sixty-one out of these 76 patients underwent FNA prior to surgery and were classified as Group 1. During the same aforementioned period, a second group of 109 patients with a parotid mass underwent an FNA, in which the cytopathologist suspected a Warthin tumor. Of these patients, 53 underwent surgical excision at our hospital. However, only 50 of the 53 were classified as Group 2, since 3 cases did not have sufficient characteristics for Warthin tumor. After re-evaluation of the pathological and cytological specimens, in order to ensure that the correct diagnosis had been made, the medical records were evaluated for demographic data, presenting symptoms, preoperative work-up, treatment, outcomes and complications.

\section{Statistics}

Initially, we analyzed Group 1. True positive (TP) was considered when correct pre-operative identification of Warthin tumor was made. Sensitivity, the proportion of actual positives correctly identified as such, was calculated. Afterwards, we analyzed Group 2. Histological identification of any malignant tumor was considered as a false negative (FN). Negative predictive value, the proportion of patients with negative test results correctly diagnosed, was also calculated.

(C) 2012 Hirshoren et al; licensee Herbert Publications Ltd. This is an open access article distributed under the terms of Creative Commons Attribution License (http:// creativecommons.org/licensesby/2.0), This permits unrestricted use, distribution, and reproduction in any medium, provided the original work is properly cited 

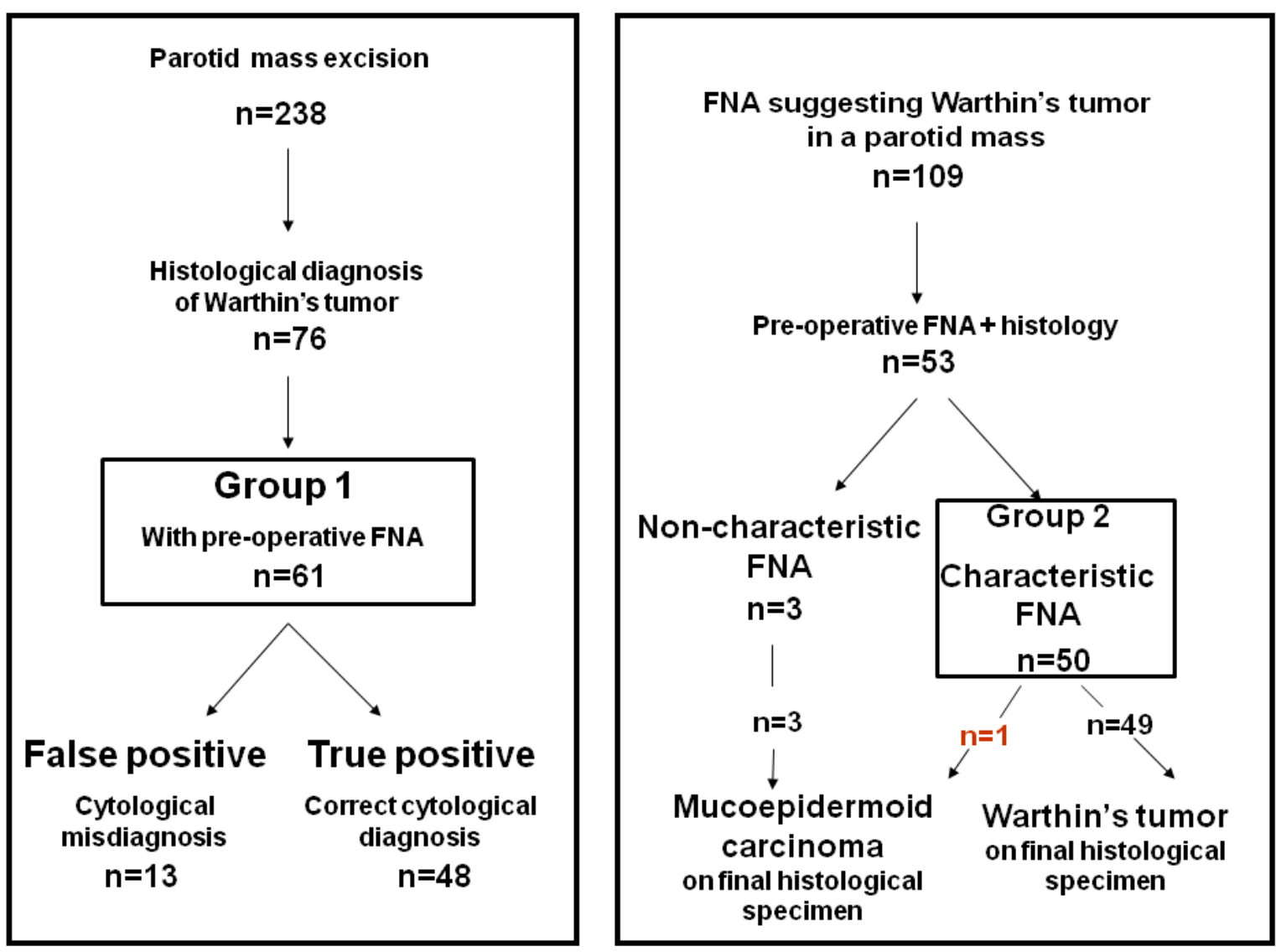

Figure 1: Schematic description of the different study groups and outcomes

\section{Results}

General (Table 1):

During the study period, 238 excised parotid gland tumors were examined in the pathology department. Of these, 75 were parotid gland Warthin tumors (31.5\%). One patient had a Warthin tumor in the submandibular gland. Overall, 76 patients had Warthin tumor, including 59 men and 17 women, with a mean age of $55.4 \pm 10.8$ years. Of these, 61 patients (group 1) had FNA done before surgery. More than half of the patients were smokers (52.6\%). The tumor presented on the right and left sides with the same frequency. Only 2 patients suffered from a bilateral disease (2.63\%). A painless facial mass was the most common presenting symptom (97.3\%). One patient presented with acute parotitis and another with facial nerve weakness. Most patients underwent imaging before surgery. CT-scan was the preferred imaging modality (84\%). All parotid gland tumors were located in the superficial lobe. Superficial parotidectomy was performed in most cases (94.7\%). The mean duration of follow-up was $90 \pm 53$ months (range 2-172 months) and there were no cases of recurrence or death from the disease. Post-operative facial nerve weakness was noted in 6 patients (8.95\%). In one patient the nerve weakness was permanent.

FNA accuracy (Figure 1):

A correct pre-operative FNA identification of a histologically proven Warthin tumor was made in 48 out of 61 patients in Group 1, i.e. a $78.7 \%$ sensitivity. In one case, an oncocytoma was suggested on the pre-operative FNA, whereas in the other 12 patients the FNA results were inconclusive.

Out of 53 FNAs reported as a Warthin tumor, in which there was subsequent histological diagnosis, 3 cases had cytological examinations lacking characteristic parameters. In all of these 
"equivocal" cases the final post-operative histology was malignant Out of the remaining 50 FNAs reported as consistent with Warthin tumor (Group 2), 1 case was confirmed later to be malignant (a negative predictive value of $98 \%$ ). Re-evaluation of this exceptional, apparently false negative case, revealed dysplastic features not considered characteristic of Warthin tumor. In all the above 4 cases the malignant tumor turned out to be a low-grade mucoepidermoid carcinoma.

\section{Discussion}

Warthin tumor, initially described in $1929^{\circ}$, is a benign tumor more common in men in their 50's, 60's and 70's. It typically presents as a slow-growing parotid mass. Squamous metaplastic cells may be identified in more than $60 \%$ of cases $[5,6]$.

There are few case reports demonstrating a malignant tumor adjacent to a benign Warthin lesion. Most of these cases were associated with previous radiotherapy [10]. Moreover, there are few case reports describing malignant transformation of Warthin tumors. Squamous cell carcinoma was the most common type of carcinoma arising within a Warthin tumor $[2,11]$ However, in most instances, it was difficult to rule out the possibility of a coincidental occurrence of Warthin tumor and a carcinoma. It is reasonable to conclude that Warthin tumor ought to be considered a benign disease. Those in favor of surgical excision do not claim the possibility of malignancy as justification for surgery, but rather, the unreliability of the preoperative FNA results.

Obviously, misdiagnosis of a malignant tumor in patients with pre-operative benign cytological_examination, falsely interpreted as Warthin tumor, is the error one needs to avoid. The question is whether this error is of practical concern in a large hospital with experienced cytopathologists. This concern has not been specifically addressed.

In a previous study, $74 \%$ of patients $(n=31)$ with a histologically confirmed Warthin tumor had the same pre-operative cytological diagnosis. The cytological misdiagnosis of the other $26 \%$ with a Warthin tumor was due to predominance of necrotic or cellular debris, epithelial metaplasia with atypia, appearance of spindle cells, and abundant mucin with keratinized squamous cells. There was no data regarding cases of FNA's falsely suggesting Warthin tumor [7]. Similar findings were presented in another study analyzing 16 patients. The cytological accuracy was $81 \%$ (3 cases had a preoperative FNA suggesting a malignant tumor) [5]. In another study,

\begin{tabular}{lll}
\hline Gender & 17 women $(25.4 \%)$ & 59 men $(74.6 \%)$ \\
Mean age & $55.4 \pm 10.8$ years & \\
follow-up & $90 \pm 53$ months & \\
bilateral disease & $2(2.63 \%)$ & \\
smokers & $40(52.6 \%)$. & \\
Post-operative facial & Transient 5 (7.4\%). & Permanent 1 (1.49) \\
\hline
\end{tabular}

Table 1: The demographic characteristics and clinical features of 76 patients with warthin tumor.
37 out of 47 patients (79\%) had a correct FNA diagnosis confirmed by histology [6]. Similar to these results, the sensitivity in our series was $78.7 \%$. In another study, 21 patients out of 32 (65\%) had a correct pre-operative diagnosis of Warthin tumor. The authors later added 40 additional cytological slides from a wide variety of parotid lesions, including malignant tumors. None of the additional malignant cytological examinations were diagnosed as Warthin tumor[8]. However, one must take into consideration that the cytopathologists were aware of the study's goals.

In our study, we demonstrate one case with so-called characteristic cytological examination and 3 other cases with ambiguous findings suggesting a Warthin tumor. All turned out to be malignant in the final histological specimens. In all cases with false negative cytological interpretation, mucoepidermoid carcinoma was diagnosed. The one case which was interpreted as consistent with a Warthin tumor was later re-evaluated, and was found to be a diagnostic error. In retrospect, this case ought to have been reported as inconclusive. Cystic changes are well-recognized as diagnostic pitfalls in the cytological assessment of salivary gland lesions. This problem is particularly associated with Warthin tumor. It is generally accepted that mucoepidermoid carcinoma presents the greatest diagnostic challenge in salivary gland FNA cytology, as many tumors are cystic, low-grade neoplasms, and exhibit deceptively bland cytological details [12].

To date, surgical excision is considered the treatment of choice in the management of Warthin tumors. Excellent outcomes have been noted, even in patients treated by limited resections[2]. However, the benign nature of Warthin tumors, even in cases in which surgery had been postponed for years due to the patients' wishes, should encourage surgeons to consider conservative treatment. Conservative management may be supported by a comprehensive study demonstrating 36 pre-operative FNA's with a diagnosis of Warthin tumor, all of which were confirmed histologically [12]. In this study, we demonstrate a large series of 53 patients with apparent Warthin tumor on FNA, who had their parotid gland removed. Since our current policy is to operate on all patients with cytological findings suggesting a Warthin tumor, we can only speculate that patients who did not undergo surgery had been treated elsewhere. In the vast majority of patients who underwent surgery at our institution, a specific and accurate pre-operative diagnosis was made by FNA. Recently, another study demonstrated a high FNA accuracy which could offer the possibility of a reliable pre-operative diagnosis of Warthin tumor [4]. We must stress out, that all cytological interpretations, both in the above study and in our own study, were carried out by experienced cytopathologists working in tertiary university hospitals, which is not valid for all medical centers.

The necessity for preoperative FNA is a debatable issue. It is considered an acceptable surgical practice to excise parotid masses without a pre-operative FNA. We would claim that this practice may be outmoded, as a pre-operative FNA may obviate the need for surgery of a Warthin tumor, which of note, is the 
second most common benign parotid tumor. In any case, cytological interpretations should be considered in the context of the history, physical examination findings, and imaging. In any case where the clinical judgment is suspicious for a malignant lesion, surgical excision is mandatory, regardless of the FNA's result.

\section{Conclusions}

Warthin tumor is a relatively common benign salivary gland lesion. The most important concern is the possibility of failure in detecting malignant tumors in the pre-operative evaluation. In cases of uncertainty, sampling errors may be reduced by US-guided reaspiration of the solid compartment adjacent to the cystic areas of the lesion. Nevertheless, one should not rely upon a borderline FNA, which does not have the classic features of Warthin tumor. Only cases with characteristic cytological features should be considered as benign Warthin lesions.

Our study has demonstrated that the majority of Warthin tumors have a characteristic cytomorphological appearance on FNA (negative predictive value of $98 \%$ ). Since the risk of malignant degeneration is known to be negligible, we recommend in selected patients observation as a reasonable alternative to surgery. Strict follow-up, including at least one repeated FNA, preferably under US guidance, is recommended for patients who do not undergo surgery.

\section{Author details}

2Department of Pathology, Hadassah Hebrew-

University Medical Center, Jerusalem, Israel.

\section{Conflict of interest}

Authors declare that they have not conflict of interest.

\section{Article history}

Received: 09-Jan-2012 Revised: 30-Jan-2012

Accepted: 15-Feb-2012 Published: 20-Mar-2012

\section{References}

1. Bell, D. et al. Warthin adenocarcinoma: analysis of $\mathbf{2}$ cases of a distinct salivary neoplasm. Ann Diagn Pathol 13, 201-207

2. Iwai, H. et al. Local excision procedure for Warthin's tumor of the parotid gland. Otolaryngol Head Neck Surg 132, 577-580

3. Reddy, V. M. et al. Conservative management of warthin tumour. J Otolaryngol Head Neck Surg 37, 744-749

4. Viguer, J. M. et al. Role of fine needle aspiration cytology in the diagnosis and management of Warthin's tumour of the salivary glands. Cytopathology 21, 164-169

5. Ballo, M. S. et al. Sources of diagnostic error in the fine-needle aspiration diagnosis of Warthin's tumor and clues to a correct diagnosis. Diagn Cytopathol 17, 230-234

6. Flezar, M. et al. Warthin's tumour: unusual vs. common morphological findings in fine needle aspiration biopsies. Cytopathology 13, 232-241

7. Parwani, A. V. et al. Diagnostic accuracy and pitfalls in fine-needle aspiration interpretation of Warthin tumor. Cancer 99, 166-171

8. Lewis, D. R. et al. Improving cytological diagnosis and surgical management of parotid adenolymphoma. Br J Surg 86, 1275-1279

9. Warthin, A. S. Papillary Cystadenoma Lymphomatosum: a Rare Teratoid of the Parotid Region. J Cancer Res 13, 116-25
10. Seifert, G. et al. Histologic subclassification of the cystadenolymphoma of the parotid gland. Analysis of $\mathbf{2 7 5}$ cases. Virchows Arch A Pathol Anat Histol 388, 13-38

11. Williamson, J. D. et al. Mucoepidermoid carcinoma involving Warthin tumor. A report of five cases and review of the literature. Am J Clin Pathol 114, 564-570

12. Stewart, C. J. et al. Fine-needle aspiration cytology of salivary gland: a review of 341 cases. Diagn Cytopathol 22, 139-146 